\title{
Auditory Brainstem Response Improvements in Hyperbillirubinemic Infants
}

\author{
Farzaneh Zamiri Abdollahi ${ }^{1}$, Tayebeh Ahmadi ${ }^{1}$, Vinaya Manchaiah ${ }^{2,3,4}$, and Yones Lotfi ${ }^{1}$ \\ ${ }^{1}$ Department of Audiology, University of Social Welfare and Rehabilitation Sciences, Tehran, Iran, \\ 2Department of Speech and Hearing Sciences, Lamar University, Beaumont, TX, USA, \\ ${ }^{3}$ Linnaeus Centre HEAD, The Swedish Institute for Disability Research, Department of Behavioral Sciences and Learning, \\ Linköping University, Linköping, Sweden, \\ ${ }^{4}$ Audiology India, Mysore, Karnataka, India
}

$\begin{array}{ll}\text { Received } & \text { September 20, 2015 } \\ \text { Revised } & \text { November 2, 2015 } \\ \text { Accepted } & \text { November 29, 2015 }\end{array}$

Address for correspondence

Tayebeh Ahmadi, $\mathrm{PhD}$

Department of Audiology,

University of Social Welfare

and Rehabilitation Sciences,

Kodakyar Ave, daneshjo Blvd, Evin,

1985713831, Tehran, Iran

Tel +98-21-222180008

Fax +98-21-22180121

E-mail tahmadi84@yahoo.com
Background and Objectives: Hyperbillirubinemia in infants have been associated with neuronal damage including in the auditory system. Some researchers have suggested that the bilirubin-induced auditory neuronal damages may be temporary and reversible. This study was aimed at investigating the auditory neuropathy and reversibility of auditory abnormalities in hyperbillirubinemic infants. Subjects and Methods: The study participants included 41 full term hyperbilirubinemic infants (mean age 39.24 days) with normal birth weight $(3,200-$ 3,700 grams) that admitted in hospital for hyperbillirubinemia and 39 normal infants (mean age 35.54 days) without any hyperbillirubinemia or other hearing loss risk factors for ruling out maturational changes. All infants in hyperbilirubinemic group had serum bilirubin level more than 20 milligram per deciliter and undergone one blood exchange transfusion. Hearing evaluation for each infant was conducted twice: the first one after hyperbilirubinemia treatment and before leaving hospital and the second one three months after the first hearing evaluation. Hearing evaluations included transient evoked otoacoustic emission (TEOAE) screening and auditory brainstem response (ABR) threshold tracing. Results: The TEOAE and $A B R$ results of control group and TEOAE results of the hyperbilirubinemic group did not change significantly from the first to the second evaluation. However, the ABR results of the hyperbilirubinemic group improved significantly from the first to the second assessment $(p=0.025)$. Conclusions: The results suggest that the bilirubin induced auditory neuronal damage can be reversible over time so we suggest that infants with hyperbilirubinemia who fail the first hearing tests should be reevaluated after 3 months of treatment.

J Audiol Otol 2016;20(1):13-16

KEY WORDS: Hyperbilirubinemia · Hearing loss · Auditory neuropathy spectrum disorder . $A B R \cdot O A E$.

\section{Introduction}

Bilirubin toxicity affects auditory neural function in a variety of ways that most of them are poorly understood. In general, the greater the central nervous system bilirubin exposure, the more widespread and severe the effect on auditory function will be. Because bilirubin exposure cannot be quan-

This is an Open Access article distributed under the terms of the Creative Commons Attribution Non-Commercial License (http://creativecommons. org/licenses/by-nc/3.0/) which permits unrestricted non-commercial use, distribution, and reproduction in any medium, provided the original work is properly cited. tified precisely, the auditory function after exposure is also quite variable, ranging from subtle auditory impairment to deafness [1]. Based on human autopsy studies cochlear function is expected to be unaffected with bilirubin toxicity [2]. The normal auditory function relies on synchronized neuronal activity. It is very likely that bilirubin toxicity would result in neuronal disorders that can range from mild dyssynchonous firing to more severe dyssynchronous firing that would make the auditory system completely nonfunctional [3]. However, it is possible to measure the functional integrity of the cochlea itself [via otoacoustic emissions (OAE) testing] inde- 
pendent of the functional integrity of the neural pathways beyond the cochlea [via auditory brainstem response (ABR)] [1].

A finding of normal cochlear function and abnormal or absent auditory neural function has been referred to as auditory neuropathy spectrum disorder (ANSD) or auditory dyssynchrony [4]. One of the earliest reports of ANSD was in children with auditory impairment associated with hyperbilirubinemia [1]. Berlin, et al. [5] reported the results of diagnosis and treatment of 260 patients with documented ANSD. Of the 150 patients with sufficient history to determine risk factors, the greatest number, 74 patients or $48.8 \%$, had a history of hyperbilirubinemia. Furthermore, some researchers have suggested that bilirubin-induced hearing loss is temporary and may also be reversible by decrement of bilirubin levels in blood $[6,7]$. The current study was aimed at investigating the $\mathrm{ABR}$ and its' changes in hyperbilirubinemic infants.

\section{Subjects and Methods}

Study participants included 80 infants, 41 infants with hyperbilirubinemia without other hearing impairment risk factors and 39 normal infants without any hearing loss risk factors. Both groups were matched in terms of age (between 30 to 45 days old) and sex. They were recruited from Akhavan Rehabilitation Center, University of Social Welfare and Rehabilitation Sciences. The mean age ( \pm standard deviation) of infants in hyperbilirubinemic group and normal group was $39.24( \pm 2.55)$ and $35.54( \pm 3.72)$ days respectively. The inclusion criteria for hyperbilirubinemic group were: full-term infants with a normal weight and appearance without any disease or other hearing loss risk factors, and natural delivery. Birth weight was between 3,200 to 3,700 grams. They were breast-fed from birth with no history of prenatal or perinatal complications, with Appearance, Pulse, Grimace, Activity, and Respiration score between 9 to 10. In addition, all infants in hyperbilirubinemic group had serum bilirubin level more than 20 milligrams per deciliter (because risk of neuronal damage is higher in infants with blood serum bilirubin level over $20 \mathrm{mg} / \mathrm{dL}$ [8]). They had undergone one exchange blood transfusion and then been receiving one phototherapy with fluorescent phototherapy device for 4-7 days. Based on blood test, in all subjects the level of bilirubin decreased after treatment.

\section{Data collection}

Hearing evaluation for each infant was conducted twice: for hyperbilirubinemic infants the first, after hyperbilirubinemia treatment and the second, 3 months after the first hearing evaluation. for normal infants the first assessment was performed within first month after birth and the second, 3 months after the first evaluation. Hearing evaluations included transient evoked otoacoustic emission (TEOAE) screening by Otodynamics ILO v6 (Otodynamics Ltd., Hatfield, United Kingdom) device and ABR threshold tracing using the Madsen-Aurical ICS Charter EP 2000 (GN Otometrics A/S, Taastrup, Denmark). Both performed in an acoustic room while infants were naturally sleep (without sedation).

At first, parents were informed about test procedures and a written consent was obtained. Following this, infants underwent TEOAE screening in both ears. Acceptable reproducibility and artifact rejection were set at $90 \%$ and $18 \%$ respectively. TEOAE results were classified into pass or fail automatically from the instrument. Subsequently, ABR testing was conducted using alternating click stimuli ( 0.1 milliseconds). Desirable electrode impedance was less than 5 kilo ohms and acceptable inter-electrode impedance was under 2 kilo ohms. ABR testing was started at $70 \mathrm{dBnHL}$. The intensity was decreased with $10 \mathrm{~dB}$ step size. ABR threshold was defined as the minimum response level at which wave $\mathrm{V}$ could be detected twice. Average threshold of the ears were categorized into three groups including: group 1) $\mathrm{ABR}$ threshold $\leq 30 \mathrm{dBnHL}$, group 2) ABR threshold between 35-65 dBnHL, and group 3) ABR threshold $\geq 70 \mathrm{dBnHL}$ ).

Data were analyzed using SPSS v16 software (SPSS Inc., Chicago, IL, USA). Kolmogorov-Smirnov test showed that data distributions were not normal. Mann-Whitney U and Wilcoxon tests were performed to compare the results between and within groups respectively.

\section{Results}

Table 1 and 2 shows TEOAEs screening and ABR test results at first and second evaluations.

At second evaluation 7 infants who had ABR threshold values between $35-65 \mathrm{dBnHL}$ at first assessment, showed $\leq 30$ $\mathrm{dBnHL}$ threshold. Infants with $\mathrm{ABR}$ threshold $\geq 70 \mathrm{dBnHL}$ at first evaluation still showed the same ABR threshold at second evaluation.

Table 1. Comparison of TEOAE results (pass/fail) between normal and hyperbilirubinemic groups from first and second evaluations

\begin{tabular}{lcccccc}
\hline & \multicolumn{2}{c}{ Normal } & & \multicolumn{2}{c}{ Hyperbilirubinemia } \\
\cline { 2 - 3 } \cline { 5 - 6 } & $\begin{array}{c}\text { Pass } \\
\text { (number of } \\
\text { infants) }\end{array}$ & $\begin{array}{c}\text { Fail } \\
\text { (number of } \\
\text { infants) }\end{array}$ & & $\begin{array}{c}\text { Pass } \\
\text { (number of } \\
\text { infants) }\end{array}$ & $\begin{array}{c}\text { Fail } \\
\text { (number of } \\
\text { infants) }\end{array}$ \\
\hline First TEOAE & 37 & 2 & & 38 & 3 \\
Second & 38 & 1 & & 38 & 3 \\
\hline
\end{tabular}

TEOAE: transient evoked otoacoustic emission 
Table 2. Comparison of the number (and percent) of infants with three ABR threshold classifications between normal and hyperbilirubinemia groups

\begin{tabular}{|c|c|c|c|c|c|c|}
\hline & \multicolumn{2}{|c|}{ ABR Thresholds $\leq 30 \mathrm{dBnHL}$} & \multicolumn{2}{|c|}{ ABR thresholds $35-65 \mathrm{dBnHL}$} & \multicolumn{2}{|c|}{ ABR thresholds $\geq 70 \mathrm{dBnHL}$} \\
\hline & Normal & Hyperbilirubinemia & Normal & Hyperbilirubinemia & Normal & Hyperbilirubinemia \\
\hline $1 \mathrm{st}$ & $37(94.9 \%)$ & $28(68.3 \%)$ & $2(5.1 \%)$ & $11(26.8 \%)$ & 0 & $2(4.9 \%)$ \\
\hline 2nd & 37 (94.9\%) & 35 (85.4\%) & $2(5.1 \%)$ & $4(9.8 \%)$ & 0 & 2 (4.9\%) \\
\hline
\end{tabular}

ABR: auditory brainstem response

Table 3. Statistical results (ABR and TEOAE) with Mann-Whitney $U$ test between normal and hyperbilirubinemia groups

\begin{tabular}{lcc}
\hline & TEOAE & ABR \\
\hline 1st evaluation & $U^{*}=780, p=0.646$ & $U=617, p=0.009$ \\
2nd evaluation & $U=780, p=0.646$ & $U=717, p=0.164$ \\
\hline
\end{tabular}

*Mann-Whitney U test. ABR: auditory brainstem response, TEO$A E$ : transient evoked otoacoustic emission

Between group comparison: Mann-Whitney U test was performed to compare the results between normal and hyperbilirubinemic groups at first and second evaluations (Table 3). At first and second evaluations, OAE test results did not show any significant difference between normal and hyperbilirubinemic groups. At the first evaluation, ABR test results were significantly different between the two groups and $\mathrm{ABR}$ threshold was higher in hyperbilirubinemic group than normal group (mean ranks of normal group and hyperbilirubinemic groups were 35.93 and 45.08 respectively). However, based on Mann-Whitney U test, at the second evaluation there were not any significant threshold difference between two groups (mean ranks in normal group and hyperbilirubinemic group were 38.43 and 42.58 respectively) as the mean rank of the hyperbilirubinemic group showed improvement from the first evaluation.

Within groups comparisons: The results of the Wilcoxon test suggested that the TEOAE and ABR results in normal group did not show any significant changes from the first to the second evaluation $(p=1.000)$. TEOAE results in the hyperbilirubinemic group did not show any significant changes from the first to the second evaluation $(p=1.000)$ but the ABR thresholds improved significantly from the first to the second assessment $(p=0.025)$.

\section{Discussion}

In the present study, there was no significant difference in TEOAE results between normal and hyperbilirubinemic groups. At the first evaluation, infants in experimental group showed more ABR abnormalities (13 out of 41 infants) than second evaluation (6 out of 41 infants). At second evaluation 7 infants who had ABR thresholds between $35-65 \mathrm{dBnHL}$ at first assessment, showed thresholds $\leq 30 \mathrm{dBnHL}$ and therefore there was no significant difference between normal and hyperbili- rubinemic group at second evaluation. Infants with thresholds $\geq 70 \mathrm{dBnHL}$ at first evaluation still remained unchanged. These results indicate that hearing loss or auditory abnormality in infants with hyperbilirubinemia can be temporary and would improve over time after proper treatment.

Previous research has shown that hyperbilirubinemia in infants is highly common and can cause abnormal ABR results [9]. Hence, ABR evaluation in hyperbilirubinemic infants is highly recommended to assess auditory nerve and auditory brainstem [10]. Although correlation between auditory disorder and bilirubin-induced encephalopathy has been studied for a long time, type and site of lesion are not exactly known. Studies have shown that in hyperbilirubinemia, different parts of auditory system can be involved including cochlea; auditory nerve; spiral ganglion; cochlear nucleus; olivary complex; and inferior colliculus $[1,8,11,12]$. Cochlear examinations with electronic microscope have not shown any significant abnormality but it does not guarantee normal cochlear function [12]. Bilirubin can influence synaptic transmissions such as construction, release and reuptake of neurotransmitters [11]. It seems that auditory thalamus and cortex are remained unaffected with hyperbilirubinemia [1]. The current study suggests that the bilirubin-induced auditory changes are at the level of lower brainstem (i.e., abnormal ABR) and not at cochlear level (i.e., normal/pass TEOAEs).

In the present study, at first evaluation, 10 infants with hyperbilirubinemia showed ANSD results (i.e., normal OAE and abnormal ABR), but ABR responses in 7 infants showed improvement at second evaluation (from $A B R$ threshold between $35-65 \mathrm{dBnHL}$ to $\leq 30 \mathrm{dBnHL}$ ). It has been suggested that infant hyperbilirubinemia can be physiologic and safe [13]. Central impairments after hyperbilirubinemia could be reversible [14] and reversible changes after hyperbilirubinemia treatments have been reported [15]. In newborn guinea pigs, one hour after bilirubin injection, compound action potential and ABR thresholds increased gradually and after 8 hours reached to a peak. After 72 hours, some consequences disappeared [16]. Similarly in the current study ABR impairments in $7(17 \%)$ infants were reversible after treatment. It can be argued that at least in some hyperbilirubinemic cases, auditory changes in central auditory system could be transient, and transient changes in brainstem is not always accompanied 
with structural changes and/or hearing loss [17]. Also, it can be inferred that ABR is a good index for evaluating bilirubininduced neurotoxicity [18].

Based on present study, we suggest that infants with hyperbilirubinemia that fail the hearing screening at first assessment should be reevaluated after treatment and before any hasty hearing intervention happened. Also it is worthwhile including both OAE and ABR testing in hearing screening of infants with hyperbilirubinemia. This would help clinicians identify infants with potential risk for auditory neuropathy disorder.

The study results strengthen the arguments of previous researchers who suggested that ABR testing following hyperbilirubinemia treatment should be part of the work-up of infants with elevated serum bilirubin levels [8].

\section{Acknowledgments}

We thank all the personnel of Audiology Clinic, Akhavan Rehabilitation Center, University of Social Welfare and Rehabilitation Sciences for their help in conducting this study.

\section{Conflicts of interest}

The authors have no financial conflicts of interest.

\section{REFERENCES}

1) Shapiro SM, Nakamura H. Bilirubin and the auditory system. J Perinatol 2001;21 Suppl 1:S52-5; discussion S59-62.

2) Haustein MD, Read DJ, Steinert JR, Pilati N, Dinsdale D, Forsythe ID. Acute hyperbilirubinaemia induces presynaptic neurodegeneration at a central glutamatergic synapse. J Physiol 2010;588(Pt 23): 4683-93.

3) Rance G. Auditory neuropathy/dys-synchrony and its perceptual consequences. Trends Amplif 2005;9:1-43.

4) Rance G, Beer DE, Cone-Wesson B, Shepherd RK, Dowell RC, King AM, et al. Clinical findings for a group of infants and young children with auditory neuropathy. Ear Hear 1999;20:238-52.

5) Berlin CI, Hood LJ, Morlet T, Wilensky D, Li L, Mattingly KR, et al. Multi-site diagnosis and management of 260 patients with auditory neuropathy/dys-synchrony (auditory neuropathy spectrum disorder). Int J Audiol 2010;49:30-43.

6) Kuriyama M, Konishi Y, Sudo M. Auditory brainstem response in hyperbilirubinemic rats: Part II. Pediatr Neurol 1991;7:375-9.

7) Ye HB, Shi HB, Wang J, Ding DL, Yu DZ, Chen ZN, et al. Bilirubin induces auditory neuropathy in neonatal guinea pigs via auditory nerve fiber damage. J Neurosci Res 2012;90:2201-13.

8) Akinpelu OV, Waissbluth S, Daniel SJ. Auditory risk of hyperbilirubinemia in term newborns: a systematic review. Int J Pediatr Otorhinolaryngol 2013;77:898-905.

9) Silva DP, Martins RH. Analysis of transient otoacoustic emissions and brainstem evoked auditory potentials in neonates with hyperbilirubinemia. Braz J Otorhinolaryngol 2009;75:381-6.

10) Hung KL. Auditory brainstem responses in patients with neonatal hyperbilirubinemia and bilirubin encephalopathy. Brain Dev 1989; 11:297-301.

11) Li CY, Shi HB, Chen ZN, Ye HB, Song NY, Yin SK. Protein kinase $A$ and $\mathrm{C}$ signaling induces bilirubin potentiation of GABA/glycinergic synaptic transmission in rat ventral cochlear nucleus neurons. Brain Res 2010;1348:30-41.

12) el Barbary A. Auditory nerve of the normal and jaundiced rat. I. Spontaneous discharge rate and cochlear nerve histology. Hear Res 1991;54:75-90.

13) Porter ML, Dennis BL. Hyperbilirubinemia in the term newborn. Am Fam Physician 2002;65:599-606.

14) Shaia WT, Shapiro SM, Spencer RF. The jaundiced gunn rat model of auditory neuropathy/dyssynchrony. Laryngoscope 2005;115:216773.

15) Boo NY, Oakes M, Lye MS, Said H. Risk factors associated with hearing loss in term neonates with hyperbilirubinaemia. J Trop Pediatr 1994;40:194-7.

16) Simmons JL, Beauchaine KL. Auditory neuropathy: case study with hyperbilirubinemia. J Am Acad Audiol 2000;11:337-47.

17) Hankø E, Hansen TW, Almaas R, Rootwelt T. Recovery after shortterm bilirubin exposure in human NT2-N neurons. Brain Res 2006; 1103:56-64.

18) Karplus M, Lee C, Cashore WJ, Oh W. The effects of brain bilirubin deposition on auditory brain stem evoked responses in rats. Early Hum Dev 1988;16:185-94. 